\title{
MAGNETOELASTIC MICROSENSORS FOR ENVIRONMENTAL MONITORING
}

\author{
Craig A. Grimes, Mahaveer K. Jain, R. S. Singh, Qingyun Cai, Andrew Mason \\ Department of Electrical Engineering, Center for Micro-Magnetic and Electronic Devices \\ 453 Anderson Hall, The University of Kentucky, Lexington, KY 40506 USA. Email: grimes@engr.uky.edu
}

\author{
Kenichi Takahata*, Yogesh Gianchandani \\ Dept. of Electrical and Computer Engineering, Wisconsin Center for Applied Microelectronics \\ 1415 Engineering Drive, The University of Wisconsin, Madison, WI 53706 USA
}

\begin{abstract}
This paper reports on the operational characteristics, and application, of a new passive wireless micro-sensor platform based on magnetoelastic materials. We have used micro-electrodischarge machining (micro-EDM) to fabricate magnetoelastic micro-sensor arrays. In response to a time varying magnetic field, amorphous ferromagnetic magnetoelastic thin films efficiently convert magnetic energy into elastic energy. The elastic waves mechanically deform the sensor which, for ribbon shaped elements, has a characteristic mechanical resonant frequency that is a function of its length, elasticity, and material density. Since the magnetoelastic material is also magnetostrictive, as the sensor mechanically deforms it generates magnetic flux that extends remotely about the device, which can be detected by a pickup coil. The remote query capability of this sensor technology enables a host of new monitoring applications including in-situ and in-vivo experiments. The bare sensor is capable of measuring ambient temperature and pressure, liquid density, liquid viscosity, and fluid flow velocity. The sensor platform can be used for chemical sensing when used in combination with mass-changing chemically responsive layers. Using an array of the magnetoelastic sensors one can determine multiple environmental conditions simultaneously.
\end{abstract}

\section{INTRODUCTION}

Surface acoustic waves and piezoelectric microsensors are widely used for measuring small changes in temperature, pressure, viscosity, and density of a medium in contact with the sensor surface [1]. These devices require a direct electrical connection between the sensor and detection electronics, limiting many in-situ and in-vivo applications.

One way to overcome the constraint of needing electrical connections with sensors is to use a magnetostrictive substrate rather than piezoelectric. Magnetostrictive magnetoelastic thick film sensors mechanically deform when subjected to a magnetic field impulse, launching elastic waves within the sensor the magnitude of which is greatest at the mechanical resonant frequency. As the sensors are magnetostrictive, the mechanical deformations launch magnetic flux that can be detected remotely by a pick up coil. The characteristic resonant frequency of the magnetoelastic sensors, which is a function of the length, density, elasticity and Poisson's ratio of the sensor material, change in response to temperature, pressure, and ambient flow rate [2].

In the past we have reported on the use of centimeter-scale magnetoelastic sensors [2-4], $\approx 4 \mathrm{~cm} \times 1 \mathrm{~cm}$ in surface area, to remotely measure temperature, pressure, liquid viscosity, glucose concentrations, and humidity over a range of meters. In this paper we report for the first time on such micro-fabricated sensors which

\footnotetext{
* On assignment from Matsushita Electric Industrial Co., Ltd., Osaka, Japan
}

are approximately $1000 x$ smaller in surface area. Our interest is in miniaturization of the sensor platform for incorporation within conventional MEMS platforms, and fabrication of micro-sensor arrays for simultaneous measurement of environmental parameters.

In the present work a miniaturized array of four magnetoelastic sensors was fabricated, using micro-electrodischarge machining (micro-EDM) [5], each of slightly different length so that their operational frequency ranges do not overlap. As the characteristic frequency of the sensor is inversely proportional to its length, ultimately the size of the sensors is limited by the ability to scale the operating frequency range of the monitoring electronics.

\section{THEORY}

For a thin, planar, ribbon-shaped sensor of length L vibrating in its basal plane the resonant frequency of the longitudinal vibrations can be given as [6]:

$$
f=\sqrt{\frac{E}{\rho}} \frac{1}{2 l}
$$

where $E$ is Young's modulus of elasticity, $\rho$ is the density of the sensor material, $l$ is the long dimension of the sensor, and we concern ourselves with only the fundamental resonant frequency due to its relatively larger amplitude.

Below the Curie temperature Young's Modulus of the magnetoelastic sensor is linear with temperature [7], hence the resonant frequency directly tracks temperature. If the sensor is stressed either elastically or in-elastically out-of-plane vibrations are induced, with the associated energy loss acting to lower the resonant (longitudinal) frequency of the sensor; as the planar surface area is relatively large, the frequency response of the sensor is found to linearly track ambient pressure [3]. Mass loading changes both the effective elasticity and density of the magnetoelastic sensor, and thereby its resonant frequency. Earlier work coating the magnetoelastic sensors with $\mathrm{TiO}_{2}$ for use as a humidity sensor [4], has shown the ability of the magnetoelastic sensors, $28 \mu \mathrm{m}$ thick, to detect differential mass loading changes of $\approx 80 \mathrm{pg} / \mathrm{mm}^{2}$ of surface area. Hence coating the sensor with a chemically responsive layer, the elasticity or density of which change with analyte concentration, enables use of the magnetoelastic sensor platform as a chemical sensor.

\section{EXPERIMENTAL RESULTS AND DISCUSSION}

Scaling the sensor platform from a centimeter to micron scale of dimensions raises several design issues, including signal to noise ratios, the frequency response of the sensors, signal damping, environmental response, packaging, etc. that we seek to address. 


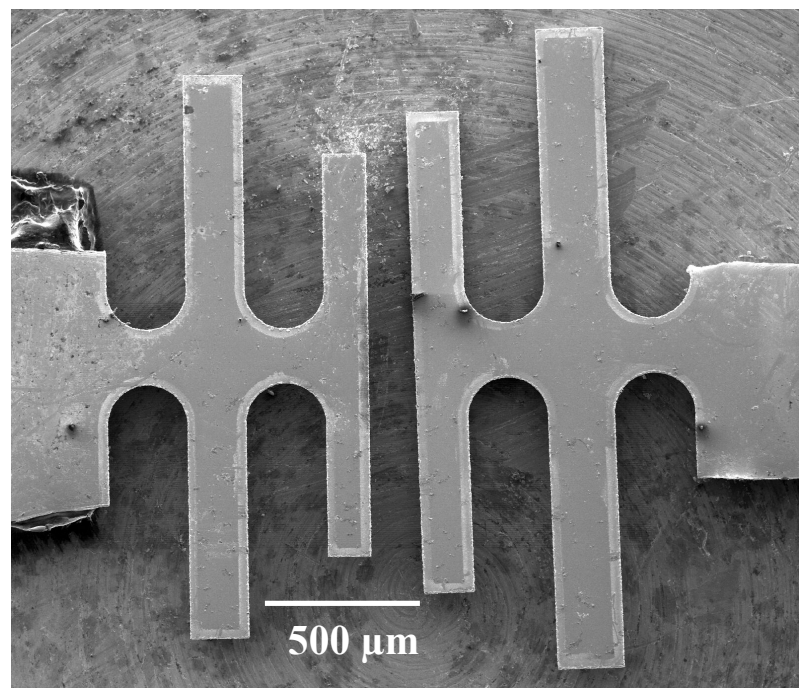

Figure 1. SEM image of four element magnetoelastic sensor array fabricated by micro-EDM.

The sensors are fabricated from $28 \mu \mathrm{m}$ thick ribbons of commercially available magnetoelastic ribbons, made by rapid melt-quenching, Metglas [6] alloys 2826MB $\left(\mathrm{Fe}_{40} \mathrm{Ni}_{38} \mathrm{Mo}_{4} \mathrm{~B}_{18}\right)$ and 2605SC $\left(\mathrm{Fe}_{81} \mathrm{~B}_{13.5} \mathrm{Si}_{3.5} \mathrm{C}_{2}\right)$. A Panasonic MG-ED72W micro-EDM machine [5] was used to define the sensor array, which is shown in Figure 1. In Figure 1, from left to right, the approximate dimensions of the sensors are: $2.2 \mathrm{~mm} \times 225 \mu \mathrm{m}, 1.6 \mathrm{~mm} \times$ $165 \mu \mathrm{m}, 1.9 \mathrm{~mm} \times 195 \mu \mathrm{m}$, and $2.5 \mathrm{~mm} \times 255 \mu \mathrm{m}$. The vibrational null point of the sensor is in the middle, hence the width of the connecting link between the two sensors, and also between sensor and supporting platform, is kept to a minimum to avoid unwanted damping of the sensors and unwanted inter-sensor coupling. The width of the supporting link for the sensor array of Figure 1 is $\approx 200 \mu \mathrm{m}$.

Figure 2 is a schematic drawing of the method used for interrogating the sensor array. A $8 \mu \mathrm{s} 7 \mathrm{Oe}$ amplitude magnetic impulse, generated by passing a current pulse down through a 5-

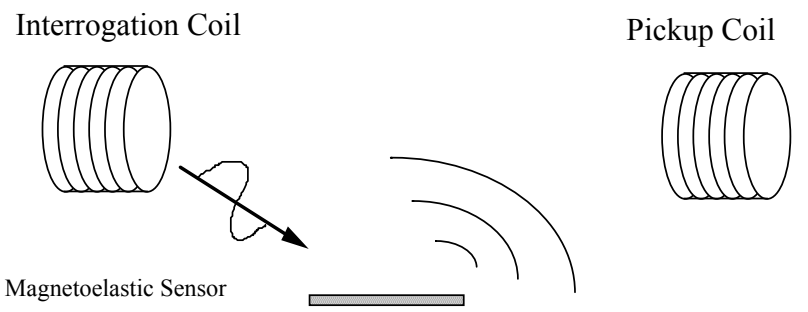

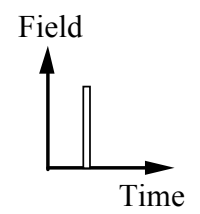

SEND

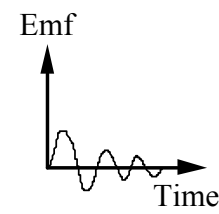

RECEIVE
Amplitude

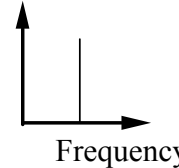

CONVERT
Figure 2. Schematic drawing illustrating remote query nature of magnetoelastic sensors.

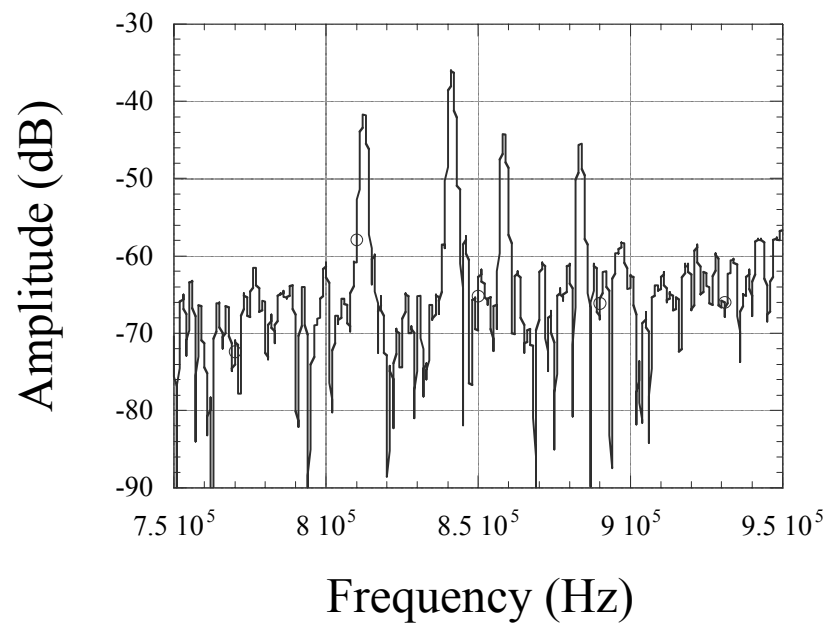

Figure 3. Frequency response of four element magnetoelastic micro-sensor array.

turn $15 \mathrm{~cm}$ diameter coil located $12 \mathrm{~cm}$ distant, is used to query the sensor array. In response to the interrogation signal the sensors oscillate emitting magnetic flux which is detected with a remotely located, $\approx 8 \mathrm{~cm}$ distant, pickup coil. A digital oscilloscope captures the output of the pickup coil. Using a Fast Fourier Transform (FFT) the oscilloscope converts the time-amplitude scale into frequency-amplitude, from which the frequency of each sensor can be readily determined. A measurement, including the time required for the FFT conversion, typically takes about $50 \mathrm{~ms}$. A typical measured frequency spectrum obtained after the FFT conversion, for the sensor array shown in Figure 1, is shown in Figure 3. A biasing de magnetic field is used to partially offset the magnetic anisotropy field of the sensor, which is a function of sensor size and alloy composition, maximizing the sensor oscillations. The lengths of the sensors are chosen such that the operational frequencies of four sensors do not overlap each other.

The magneto-mechanical coupling coefficient [9] of the sensor plays an important role in determining the amplitude of the detected signal, and changes with applied dc magnetic field. The resonant frequency of the sensor also varies with dc biasing field due to the $\Delta \mathrm{E}$ effect [10]. To maximize the amplitude of the detected signal the response of the sensor is measured as a function of dc magnetic biasing field. Figure 4 shows, for a $1.9 \mathrm{~mm} \mathrm{x}$

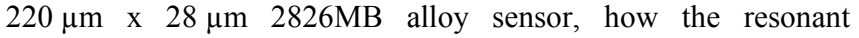
frequency and detected amplitude varies with applied dc magnetic field. The highest amplitude is obtained at a biasing field value of 8.6 Oe. However at this biasing field value the resonant frequency significantly varies with a slight deviation in magnetic field, leading to unacceptable measurement errors. Therefore the sensor array was operated using a 20.2 Oe biasing field, at which point the resonant frequency was not found to significantly vary with a \pm 0.5 Oe variation in field as can be expected due to the sensor moving through earth's field.

The biasing field is easily applied through adjacent location of a magnetically hard strip of material such as a thick-film ferrite. The magnitude of the biasing field scales with sensor dimension proportionally to the demagnetizing field. For $28 \mu \mathrm{m}$ thick sensors, typical biasing field amplitudes are 6-7 Oe for $4 \mathrm{~cm} \times 1 \mathrm{~cm}$ sensors, $\approx 23$ Oe for $2.5 \mathrm{~mm} \times 250 \mu \mathrm{m}$ sensors, and 75 Oe for $500 \mu \mathrm{m} \times$ $50 \mu \mathrm{m}$ sensors. The needed amplitude of the interrogation impulse 
also increases with sensor miniaturization; for centimeter-length sensors interrogation pulses of approximately 3 Oe are required, for millimeter-length sensors interrogation pulses of $\approx 8$ Oe are needed, while for micron-sized sensors an impulse amplitude of $\approx 12$ Oe is necessary.

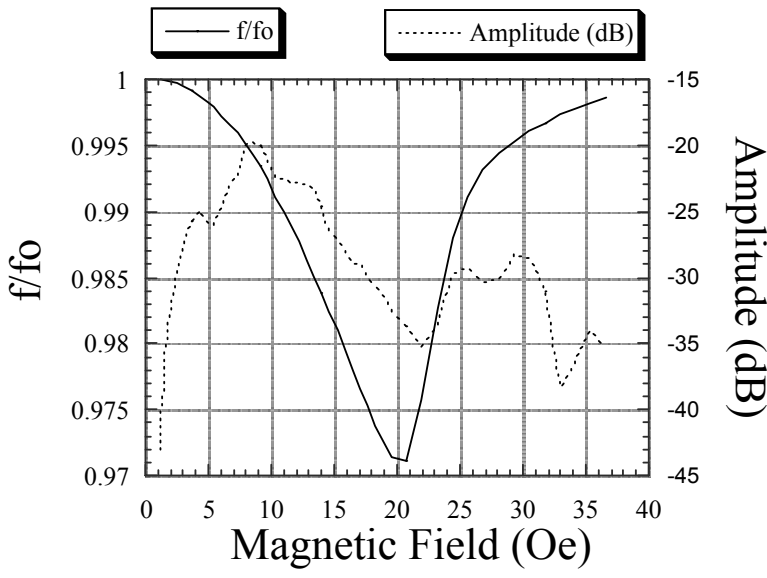

Figure 4. Relative resonant frequency and signal amplitude as a function of applied dc magnetic field for 2826 sensor ribbon.

For a given alloy, all sensors have the same optimal value of internal biasing field. The internal magnetic field is dependent upon the magnetic permeability of the sensor material, and on the demagnetizing field of the sensor. The demagnetizing field, in turn, depends on the shape and size of the sensor and the saturation magnetization of the alloy. In this work the size of the sensors have been tailored to achieve the same internal magnetic field. For that reason, as seen in Figure 1, the width of the sensor is appropriately scaled to achieve the same optimal operating field value for all sensors. For sensor arrays comprised of different magnetoelastic alloys, the use of adjacently located magnetically hard strips of different stray-field strength enables them to be independently biased with their respective optimal operating field.

The two Metglas alloys used in this work have different elasticity-temperature profiles, enabling an absolute measurement of temperature in, for example, a changing pressure environment. Recent work [3] has reported on how the pressure sensitivity of a magnetoelastic sensor can be controlled by introducing strain into the sensor. Therefore stressed and un-stressed sensors of the $2826 \mathrm{MB}$ alloy are used to measure pressure in a changing temperature environment.

Figure 5 demonstrates how temperature can be measured in a changing pressure environment by comparing the frequency response of a $2826 \mathrm{MB}$ and $2605 \mathrm{SC}$ alloy sensor. Relative frequency values are used since each sensor has an operational frequency range outside that of the other sensors. The frquencytemperature responses are linear, and higher for 2605SC in comparison to the $2826 \mathrm{MB}$ material. A linear frequency dependence is also seen for changes in pressure, an effect clearly delineated in Figure 6 which shows the frequency response of stressed and unstressed $2826 \mathrm{MB}$ alloy sensors. The pressure sensitivity of a sensor can be enhanced introducing a known amount of strain into the sensor, resulting in out of plane vibrations the dampening effect of which is directly proportional to pressure.

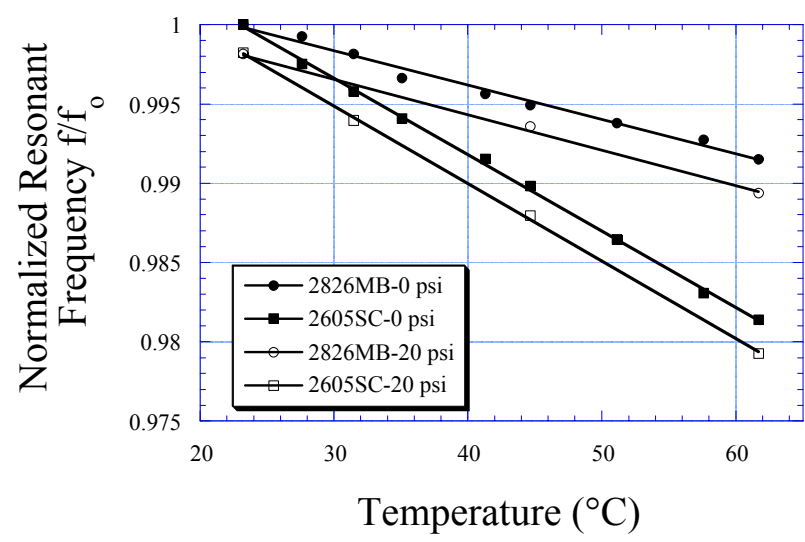

Figure 5. Absolute measurement of temperature in a changing pressure environment, through monitoring the relative frequency change in magnetoelastic sensors comprised of different alloys with the same stress levels.

Application of an additional mass $m^{\prime}$ to the mass $M$ of the sensor corresponds to an increase of its density by a factor $1+m^{\prime} / M$, which in turn changes the resonant frequency by a factor $\left(1+m^{\prime} / M\right)^{-0.5}$. If the mass increase is small compared to the mass of the sensor the shift in the resonant frequency is given by [2] $\Delta f=-f m^{\prime} / 2 M$. The frequency shifts linearly downward with increasing mass. Hence wireless, chemical sensors can be made by coating the magnetoelastic sensor with a mass-changing, chemically responsive layer. To demonstrate this a magnetoelastic $\mathrm{CO}_{2}$ sensor was made by dip-coating an amide-functionalized polymer onto a $2826 \mathrm{MB}$ micro-sensor. The polymer was synthesized from a 1:1 mole ratio of acrylamide to isooctyl acrylate, for which a $0.7 \%$ change in atmospheric $\mathrm{CO}_{2}$ concentration can be detected. Remote query monitoring of $\mathrm{CO}_{2}$ by an inexpensive, disposable sensor would find utility in a variety of applications including, for example, monitoring for food spoilage from within opaque, sealed food packages or medicine containers.

The $\mathrm{CO}_{2}$ responsive polymer consists of hydrophobic isooctylacrylate and hydrophilic acrylamide. Acrylamide provides the amide group that is sensitive to carbon dioxide due to hydrogen

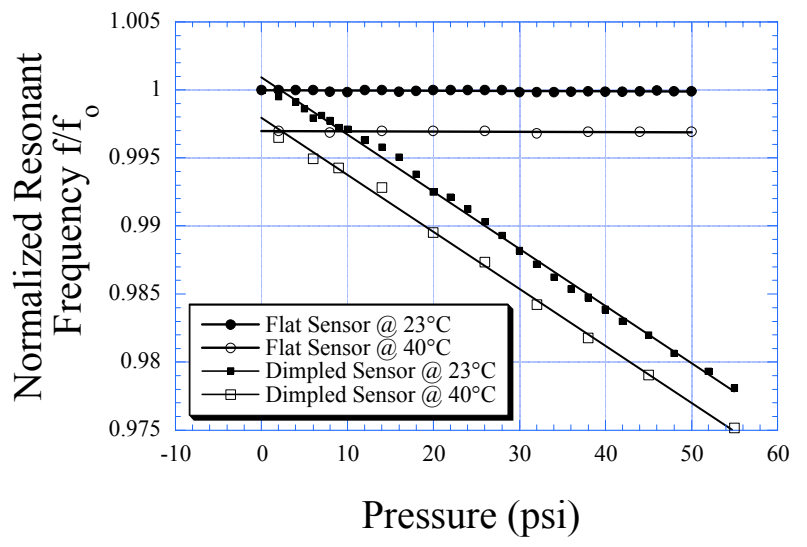

Figure 6. Absolute measurement of pressure in a changing temperature environment, through monitoring the relative frequency change in two magnetoelastic sensors comprised of the same alloys but having different stress levels. 
bonding interaction between amide and the oxygen atoms of $\mathrm{CO}_{2}$ [11]. The hydrophobically-modified copolymer, poly(acrylamideco-isooctylacrylate) was synthesized by free radical copolymerization of acrylamide and isooctylacrylate in dimethylformamide with an initial mole ratio of acrylamide to isooctylacrylate of $1: 1$ at an overall monomer concentration of 3.5 $\mathrm{mol} / \mathrm{L}$ at $75^{\circ} \mathrm{C}$, with $0.4 \mathrm{~mol} \%$ AIBN added as initiator. The solution was deoxygenated by bubbling nitrogen for an hour prior to reaction and the reaction was carried out under nitrogen atmosphere. The polymeric residue was washed in ethanol repeatedly to remove the dimethylformamide solvent and unreacted components. The polymer was applied by dip-coating in a $5 \mathrm{w} \%$ tetrahydrofuran solution. The polymer-coated sensor was dried in a vacuum oven at $80^{\circ} \mathrm{C}$ overnight to remove the solvent.

The sensor was set in the middle of a $2 \times 2 \times 6 \mathrm{~cm}$ gas-flow controlled plastic test chamber that was placed between a pair of $40 \mathrm{~cm}$ diameter Helmholtz coils used to produce the magnetic query field. The test atmosphere were generated by mixing known volumes $99 \% \mathrm{CO}_{2}$ and $99 \% \mathrm{~N}_{2}$ directly from a compressed cylinder. All experiments were performed at a room temperature of $24 \pm 1{ }^{\circ} \mathrm{C}$, and at a flow rate of $200 \mathrm{~mL} / \mathrm{min}$. The resonant frequency of the sensor was measured as it was switched between the $\mathrm{N}_{2}$ and test atmospheres containing dry $\mathrm{CO}_{2}(\mathrm{RH} 0.6 \%$ ).

Figure 7 shows the shift in the measured resonant frequency of a magnetoelastic $\mathrm{CO}_{2}$ sensor cycled between a pure $\mathrm{N}_{2}$ environment and one with $25 \% \mathrm{CO}_{2}$; the polymer coating is $2 \mu \mathrm{m}$ thick on each side of the sensor. The response profile was highly reproducible over replicate exposures, with an average standard deviation in the measurement of $3.0 \%$.

When switched from nitrogen to a carbon dioxide containing atmosphere the sensor shows a sharp drop (within 2 minutes) in the measured resonant frequency, which then recoils to a higher frequency which is then stable after a few minutes. Real time, inline monitoring of the polymer mass indicates that the sharp drop in the resonant frequency of the sensor is due to the polymer rapidly increasing in mass from absorption of $\mathrm{CO}_{2}$. The average polymer mass increase due to $\mathrm{CO}_{2}$ absorption is $2 \%$. The recoil seen in the measured resonant frequency after the initial drop is not due to mass change, which stays constant, but is most likely caused by polymer swelling driven by the $\mathrm{CO}_{2}$ diffusion. Polymer swelling increases the elasticity of the polymer which, as seen in Eq. 1, increases the resonant frequency of the sensor. The recoil seen in Figure 2 was obtained for measurements at all $\mathrm{CO}_{2}$ and humidity levels. Over the period of a month the sensor was measured twelve times to provide a measure of sensor reproducibility. The standard deviation of the measured responses was $3.8 \%$ with no trends observed over time.

\section{CONCLUSIONS}

In this paper we demonstrate the use of magnetoelastic microsensors for environmental sensing, with the simultaneous wireless measurement of temperature and pressure, and demonstration of chemical sensing by combination of the magnetoelastic sensors with a $\mathrm{CO}_{2}$ responsive polymer. The magnetoelastic sensors are passive, responding to the query field, hence could be used for long-term environmental monitoring applications where battery life is an issue. The wireless nature of the sensor platform makes it ideal for for in-situ monitoring applications.

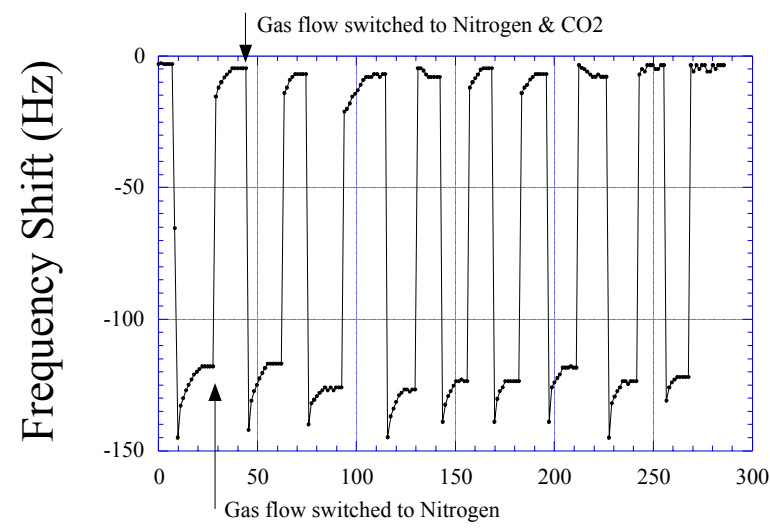

Total Elapsed Time (minutes)

Figure 7. The change in resonant frequency of the magnetoelastic $\mathrm{CO}_{2}$ sensor as it is repeatedly cycled between an atmosphere of nitrogen, and nitrogen with $25 \%$ carbon dioxide.

\section{ACKNOWLEDGEMENTS}

This work was supported by the National Science Foundation under grants ECS-9988598 and ECS-9875104, and the Department of Energy under grant DE-FG02-91ER7566.

\section{REFERENCES}

1. D. S. Ballantine, R. M. White, S. J. Martin, A. J. Ricco, G. C. Frye, E. T. Zellers, H. Wohltjen, Acoustic Wave Sensors: Theory, Design, and Physicochemical Applications, Academic Press, Boston, MA, 1997.

2. C.A. Grimes, K.G. Ong, K. Loiselle, P.G. Stoyanov, D. Kouzoudis, Y. Liu, C. Tong, "Magnetoelastic Sensors For Remote Query Environmental Monitoring", Smart Materials and Structures, 8, 639 (1999).

3. D. Kouzoudis, C.A. Grimes, "The Frequency Response of Magnetoelastic Sensors to Stress and Atmospheric Pressure," in press, Smart Materials \& Structures, 9, 2000.

4. C.A. Grimes, D. Kouzoudis, E.C. Dickey, D. Qian, et. al "Magnetoelastic Sensors in Combination With Nanometer-Scale Honeycombed Thin Film Ceramic TiO2 For Remote Query Measurement Of Humidity", J. Appl. Phys, 87, 5341 (2000).

5. T. Masaki, K. Kawata, T. Masuzawa, "Micro Electro-Discharge Machining and Its Applications", Proc. IEEE MEMS, 1990, pp. 2126

6. L.E. Kinsler and A.R. Fray, "Fundamentals of Acoustics". $3^{\text {rd }}$ ed. Ch. 3, Wiley (1983).

7. T. Shinoda, N. Soga, t. Hanada, S. Tanabe, "Young's modulus of RF-sputtered amorphous thin films in the $\mathrm{SiO}_{2}-\mathrm{Y}_{2} \mathrm{O}_{3}$ system at high temperature," Thin Solid Films, vol. 293, pp. 144-148 (1997).

8. Metglas ${ }^{\mathrm{TM}}$ is a trademark of the Honeywell Corporation.

9. T. de Lacheisserie, "Magnetoelastic properties of amorphous alloys,” J. Mag. Mag. Mat., vol. 25, pp. 251-270 (1982).

10. P. M. Anderson III, "Magnetomechanical coupling, DE effect, and permeability in FeSiB and FeNiMoB alloys," J. Appl. Phys., vol. 53, pp. 8101-8103 (1982).

11. A. Oprea, K. Henkel, R. Oehmgen, G. Appel, D. Schmeisser, H. Lauer, and P. Hausmann, "Increased sensor sensitivities obtained by polymer-coated quartz microbalances," Materials Science \& Engineering C, vol. 8-9 (1999) 509-512. 